\title{
Exploring the phase diagram of finite density QCD at low temperature by the complex Langevin method
}

\author{
Yuta Ito*广 \\ High Energy Accelerator Research Organization (KEK), \\ 1-1 Oho, Tsukuba, Ibaraki 305-0801, Japan \\ E-mail: vitoepost.kek.jp \\ Hideo Matsufuru \\ High Energy Accelerator Research Organization (KEK), \\ 1-1 Oho, Tsukuba, Ibaraki 305-0801, Japan \\ E-mail: hideo.matsufurudkek.jp

\section{Jun Nishimura} \\ High Energy Accelerator Research Organization (KEK), \\ 1-1 Oho, Tsukuba, Ibaraki 305-0801, Japan, and \\ Graduate University for Advanced Studies (SOKENDAI), \\ 1-1 Oho, Tsukuba 305-0801, Japan \\ E-mail: Gnishiepost.kek.jp
}

\section{Shinji Shimasaki}

Research and Education Center for Natural Sciences, Keio University, Hiyoshi 4-1-1, Yokohama, Kanagawa 223-85 21, Japan E-mail: Shinji.Shimasakidkeio.jp

\section{Asato Tsuchiya}

Department of Physics, Shizuoka University, 836 Ohya, Suruga-ku, Shizuoka 422-8529, Japan

E-mail: tsuchiva.asatodshizuoka.ac.jp

\section{Shoichiro Tsutsui}

High Energy Accelerator Research Organization (KEK),

1-1 Oho, Tsukuba, Ibaraki 305-0801, Japan

E-mail: stsutsuiepost.kek.jp

Monte Carlo studies of QCD at finite density suffer from the sign problem, which becomes easily uncontrollable as the chemical potential $\mu$ is increased even for a moderate lattice size. In this work we make an attempt to approach the high density low temperature region by the complex Langevin method (CLM) using four-flavor staggered fermions with reasonably small quark mass on a $8^{3} \times 16$ lattice. Unlike the previous work on a $4^{3} \times 8$ lattice, the criterion for correct convergence is satisfied within a wide range of $\mu$ without using the deformation technique. In particular, the baryon number density exhibits a plateau behavior consistent with the formation of eight baryons, and it starts to grow gradually at some $\mu$.

The 36th Annual International Symposium on Lattice Field Theory - LATTICE2018

22-28 July, 2018

Michigan State University, East Lansing, Michigan, USA.

\footnotetext{
* Speaker.

${ }^{\dagger}$ KEK-TH/2087
} 


\section{Introduction}

In finite density QCD at low temperature and high density, it is conjectured that there are various interesting phases such as the quark matter phase, the color-superconductor phase and so on. Exploring these phases based on full QCD simulation is important not only for purely academic reasons but also for understanding the interior structure of neutron stars. However, standard Monte Carlo methods face with the sign problem, which has been hindering the development in this direction so far.

The complex Langevin method (CLM) [U, [1] has been studied as a promising approach to this problem. The idea is to extend the stochastic quantization based on the Langevin equation to the cases with a complex action by complexifying the dynamical variables, where holomorphicity has to be respected in defining the drift term and the observables. The biggest problem of this approach is that the obtained results are not guaranteed to be correct. However, in recent years the reasons

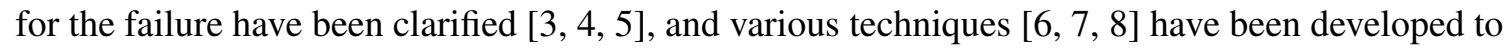
extend the applicability of this method. Thanks to these developments, finite density QCD in the

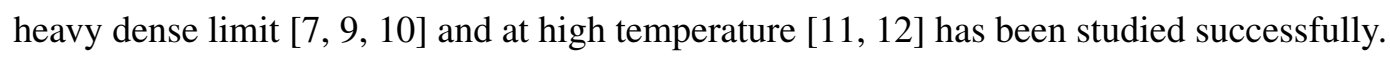

We attempt to extend this success to the low temperature region with reasonably small quark mass, where the transition to the nuclear matter phase and subsequently to the quark matter phase at larger chemical potential $\mu$ is anticipated. Extending the previous work [피] on a $4^{3} \times 8$ lattice, we perform simulations with four-flavor staggered fermions on a $8^{3} \times 16$ lattice with quark mass $m=0.01$ and $m=0.05$. (See refs. [14] for related work with two-flavor staggered fermions.) Rather surprisingly, we find that the criterion for correct convergence [5] is satisfied within a wide range of $\mu$ without using the deformation technique [ [ $]$ ] unlike the previous work [[13]. In particular, the baryon number density exhibits a plateau as a function of $\mu$, which is consistent with the formation of eight baryons, and it starts to grow gradually at some $\mu$. We obtain similar results for the two values of the quark mass although the plateau behavior becomes clearer for $m=0.01$.

We also simulate the phase quenched model with the same set of parameters using the standard RHMC algorithm. Here we obtain quite different results for the two values of the quark mass $m$ unlike the results for the full model. At $m=0.01$, the baryon number density exhibits a different plateau with lower height at smaller values of $\mu$, which is consistent with the formation of four mesons considering that $\mu$ in the phase quenched model actually corresponds to the isospin chemical potential. Thus we find a clear difference between the results of the CLM and those for the phase quenched model at $m=0.01$. At $m=0.05$, on the other hand, the baryon number density behaves quite similarly for the two models.

The rest of this paper is organized as follows. In section $\square$ we explain briefly how we apply the CLM to finite density QCD and how we judge the validity of the results. In section B we show the results obtained by the CLM and compare them with the results for the phase quenched model. The section $⿴$ is devoted to a summary and discussions.

\section{Complex Langevin method for finite density QCD}

In this paper we study finite density QCD with $N_{\mathrm{f}}=4$ staggered fermions. The partition 
function is given as

$$
Z=\int d U \operatorname{det} M[U, \mu] e^{-S_{\mathrm{g}}[U]},
$$

where $U_{x, v} \in \mathrm{SU}(3)$ are the link variables and $S_{\mathrm{g}}[U]$ is the plaquette action defined by

$$
S_{\mathrm{g}}[U]=-\frac{\beta}{6} \sum_{x} \sum_{v \neq \rho} \operatorname{tr}\left(U_{x, v} U_{x+\hat{v}, \rho} U_{x+\hat{\rho}, v}^{-1} U_{x, \rho}^{-1}\right) .
$$

The determinant $\operatorname{det} M[U, \mu]$, which is obtained by integrating out the fermions, is complex when the chemical potential $\mu$ is nonzero. This makes the standard Monte Carlo simulation difficult due to the sign problem, which becomes severer as $\mu$ is increased.

In order to overcome this problem, we apply the CLM, which is a complex extension of the stochastic quantization based on the Langevin equation. In this method, the link variables $U_{x, v}$ are complexified as $\mathscr{U}_{x, v} \in \mathrm{SL}(3, C)$, and accordingly the drift term and the observables, which are functions of $U_{x, v}$, have to be extended to functions of $\mathscr{U}_{x, v}$ holomorphically. The complexified link variables are updated according to the complex version of the Langevin equation

$$
\mathscr{U}_{x, v}(t+\varepsilon)=\exp \left[i\left(-\varepsilon v_{x, v}(\mathscr{U}(t))+\sqrt{\varepsilon} \eta_{x, v}(t)\right)\right] \mathscr{U}_{x, v}(t),
$$

where $t$ is the discretized Langevin time and $\varepsilon$ is the stepsize. The drift term $v_{x, v}(\mathscr{U})$ in eq. ([2.3) is defined by the holomorphic extension of

$$
v_{x, v}(U)=\left.\sum_{a} \lambda_{a} \frac{d}{d \alpha} S\left(e^{i \alpha \lambda_{a}} U_{x, v}\right)\right|_{\alpha=0},
$$

where $S[U]=S_{\mathrm{g}}[U]-\ln \operatorname{det} M[U, \mu]$ and $\lambda_{a}(a=1, \cdots, 8)$ are the $\mathrm{SU}(3)$ generators normalized by $\operatorname{tr}\left(\lambda_{a} \lambda_{b}\right)=\delta_{a b}$. The noise term $\eta_{x, v}(t)$ in eq. (2.3), which are $3 \times 3$ traceless Hermitian matrices, are generated with the Gaussian distribution $\exp \left(-\frac{1}{4} \operatorname{tr}\left\{\eta_{x, v}^{2}(t)\right\}\right)$.

The expectation value of an observable $O(U)$ can be obtained as

$$
\langle O(\mathscr{U})\rangle=\lim _{T \rightarrow \infty} \frac{1}{T} \int_{t_{0}}^{t_{0}+T} d t\langle O(\mathscr{U}(t))\rangle_{\eta}
$$

where the expectation value $\langle\cdot\rangle_{\eta}$ appearing on the right-hand side should be taken with respect to the Gaussian noise $\eta$, and $t_{0}$ should be sufficiently large to achieve thermalization. The effect of the complex fermion determinant is included in the complex Langevin process ([2.3]) through the drift term, and there is no need for reweighting unlike the path-deformation approach such as the generalized Lefschetz-thimble method.

It is known that the CLM fails to yield correct results in some cases. However, we can judge whether the obtained results are correct or not in the following way [ []]. Let us define the magnitude of the drift term as

$$
v=\sqrt{\frac{1}{3} \max _{x, v} \operatorname{tr}\left(v_{x, v}^{\dagger} v_{x, v}\right)}
$$

and consider its probability distribution $p(v)$. If $p(v)$ falls off exponentially or faster, the result is reliable. A slower fall-off such as a power law can be caused either by the excursion problem [B] or the singular-drift problem [䧃]. The first problem occurs when the link variables $\mathscr{U}_{x, v}$ become 

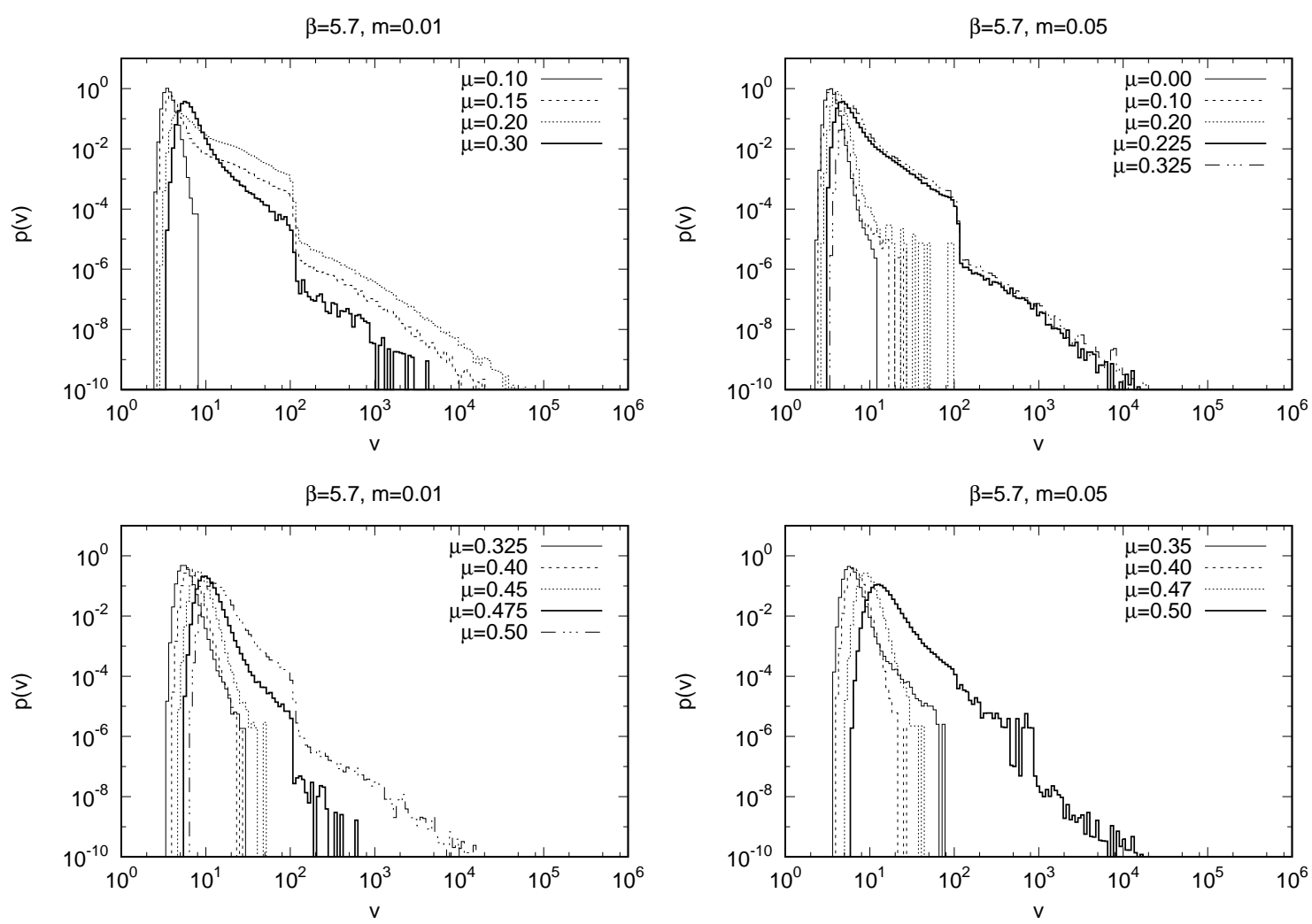

Figure 1: The probability distribution $p(v)$ of the drift term are plotted for various $\mu$ with $m=0.01$ (Left) and $m=0.05$ (Right).

too far from SU(3) matrices, and the second one occurs when the Dirac operator has near-zero eigenvalues frequently because the drift term involves the inverse of the Dirac operator.

In order to suppress the excursion problem as much as possible, we perform the gauge cooling [] as follows. (See ref. [[]] for justification.) Let us define the unitarity norm as

$$
\mathscr{N}=\frac{1}{12 N_{\mathrm{V}}} \sum_{x, v} \operatorname{tr}\left(\mathscr{U}_{x, v}^{\dagger} \mathscr{U}_{x, v}-\mathbf{1}\right),
$$

where $N_{\mathrm{V}}$ is the number of lattice sites. This quantity measures how far $\mathscr{U}_{x, v}$ is from SU(3) configurations, and it vanishes if and only if all of $\mathscr{U}_{x, v}$ are unitary matrices. After updating $\mathscr{U}_{x, v}$ by the complex Langevin equation ([2.3]), we perform a complexified gauge transformation

$$
\mathscr{U}_{x, v} \rightarrow g_{x} \mathscr{U}_{x, v} g_{x+\hat{v}}^{-1}, \quad \text { where } g_{x} \in \operatorname{SL}(3, C)
$$

in such a way that the unitarity norm is minimized.

\section{Results}

We have performed simulations on a $8^{3} \times 16$ lattice with $\beta=5.7$ and the quark mass $m=0.01$ and 0.05 . The Langevin stepsize is chosen initially as $\varepsilon=10^{-4}$, which is reduced adaptively 
when the magnitude of the drift exceeds certain threshold [ [6]. We have made $(5 \sim 15) \times 10^{5}$ total Langevin steps for each set of parameters.

Let us first check the validity of the results obtained by the CLM. In Fig. $($ (Left), the probability distribution $p(v)$ of the drift term is plotted for various $\mu$ with $m=0.01$. From these plots, we find that $p(v)$ for $\mu=0.1$ and $0.325 \leq \mu \leq 0.475$ shows a clear exponential fall-off and therefore the simulations are reliable. On the other hand, $p(v)$ for $0.15 \leq \mu \leq 0.3$ and $\mu=0.5$ shows a power law fall-off, which is actually caused by the contribution from the fermion determinant. Thus, we find that the singular-drift problem occurs in these parameter regions. Similarly, in Fig. $\square$ (Right), we show the probability distribution $p(v)$ for various $\mu$ with $m=0.05$, from which we find that the results for $0 \leq \mu \leq 0.2$ and $0.35 \leq \mu \leq 0.47$ are reliable. As in the $m=0.01$ case, the power law fall-off seen in these plots is due to the singular-drift problem.

Fig. $\square$ shows the baryon number density

$$
\langle n\rangle=\frac{1}{3 N_{V}} \frac{\partial}{\partial \mu} \ln Z
$$

obtained by the CLM for $m=0.01$ (Left) and $m=0.05$ (Right), where we plot only the reliable data judging from the probability distribution of the drift term. We also plot the RHMC results for the phase quenched model for comparison.
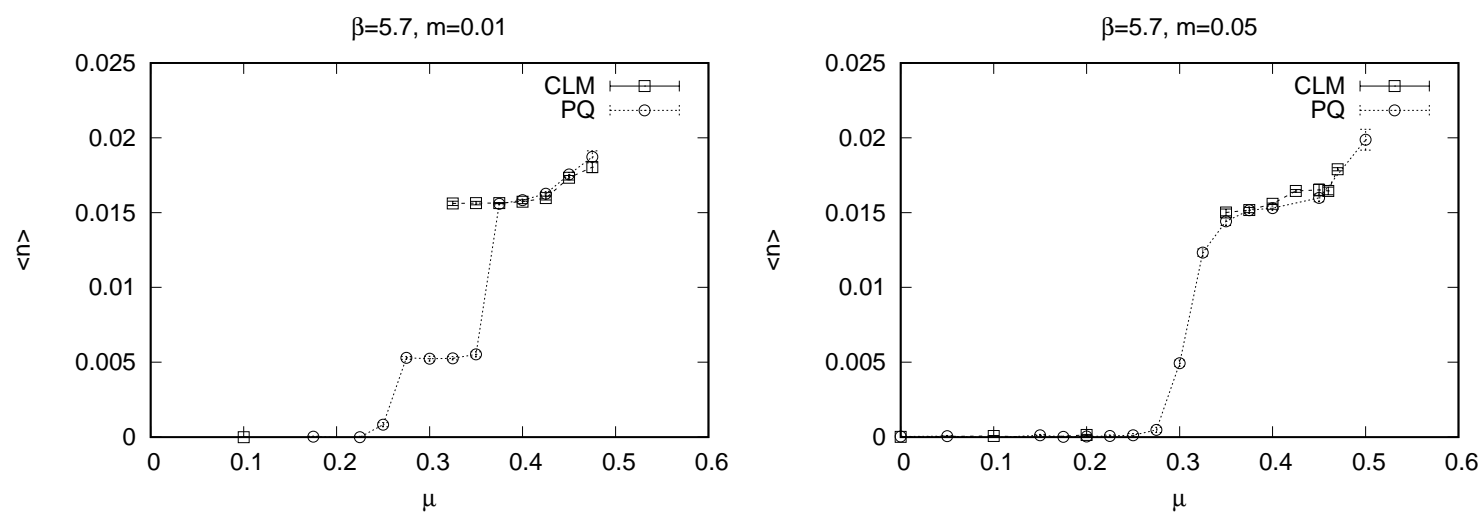

Figure 2: The baryon number density is shown as a function of the chemical potential $\mu$ for $m=0.01$ (Left) and $m=0.05$ (Right). The squares represent the CLM results which are reliable judging from the probability distribution of the drift term. The circles represent the RHMC results for the phase quenched model.

As far as we can see from the reliable data, the results obtained by the CLM do not depend much on the quark mass. Most strikingly, we observe a clear plateau for $m=0.01$ in the region $0.325 \lesssim \mu \lesssim 0.425$, which is visible for $m=0.05$ as well although it is less clear. We can also see some tendency of $\langle n\rangle$ starting to grow gradually at larger $\mu$. On the other hand, the results for the phase quenched model depend drastically on the quark mass unlike the full model results. In particular, for $m=0.01$ we observe a lower plateau at $0.275 \lesssim \mu \lesssim 0.35$, which does not appear for $m=0.05$.

In order to understand these results, it is important to note that the lattice spacing for $\beta=5.7$ and $m=0.01$ with $N_{\mathrm{f}}=4$ staggered fermions is obtained as $a \sim 0.045 \mathrm{fm}$, while it is slightly larger for $m=0.05$. Therefore the physical spatial extent of our lattice is much smaller than the QCD 
scale and the physical temperature is actually quite high. The observed behavior which mimics that in the low temperature regime in a large volume may be understood as a result of the temperature being lower than the scale of the spatial extent.

Another important thing to note is that the phase quenched model may be regarded as a full model with $\mu$ being interpreted as the isospin chemical potential due to $\operatorname{det} D(-\mu)=(\operatorname{det} D(\mu))^{*}$, and in that case $\langle n\rangle$ represents $\langle n\rangle=\left\langle\left(n_{\mathrm{u}}-n_{\mathrm{d}}\right) / 3\right\rangle$, where $n_{\mathrm{u}}$ and $n_{\mathrm{d}}$ represent the "up-quark" density and the "down-quark" number density, respectively.

The plateau in the phase quenched model may therefore be identified as a state with 4 mesons, which corresponds to $\langle n\rangle=4 \times 2 / 3 / 8^{3}=0.0052$. The reason why we observe the plateau instead of smooth increase of the isospin density as is typically observed in previous work [U5] is presumably due to the small physical volume of our lattice. The plateau in the full model observed by the CLM, on the other hand, may be identified as a state with 8 baryons, which corresponds to $\langle n\rangle=8 / 8^{3}=$ 0.0156 . The density of this state is much higher than what one would expect for the nuclear matter, but this may be also due to the severe finite volume effects.
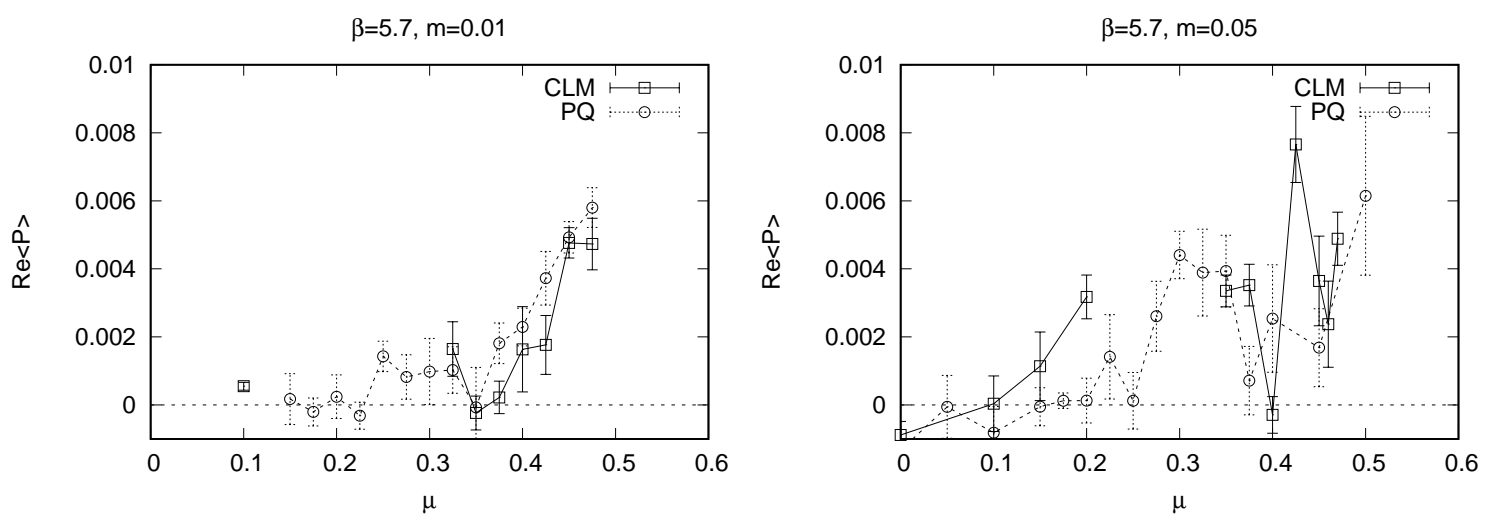

Figure 3: The expectation value of the Polyakov loop is plotted against $\mu$ for $m=0.01$ (Left) and $m=0.05$ (Right). The squares represent the CLM results which are reliable judging from the probability distribution of the drift term. The circles represent the RHMC results for the phase quenched model.

In Fig. [] we plot the expectation value of the Polyakov loop, which is found to be small at $\mu \lesssim 0.45$ for both models with $m=0.01$ and $m=0.05$ despite the high temperature. This is considered as a consequence of the small spatial extent of our lattice.

\section{Summary and discussions}

In this paper we have made an attempt to extend the success of the CLM in finite density QCD to the lower temperature region with reasonably small quark mass using four-flavor staggered fermions on a $8^{3} \times 16$ lattice. The physical size of our lattice is small due to the choice $\beta=5.7$, which is motivated by the necessity to avoid the excursion problem [एप]]. With that in mind, our results can be summarized as follows.

First we have investigated the validity of the CLM based on the probability distribution of the drift term. Interestingly, there is a region in which the CLM works even at large $\mu$. This is in contrast to the situation of the previous work on a $4^{3} \times 8$ lattice [[13]], where the use of the 
deformation technique [ [8] was necessary to avoid the singular-drift problem in the large $\mu$ region. In fact, when $\mu$ is chosen within the region of validity of the CLM, the eigenvalue distribution of the Dirac operator exhibits a gap along the real axis, and therefore the singular-drift problem does not occur.

Second the baryon number density exhibits a plateau as a function of the chemical potential, which may be identified as the formation of the nuclear matter. The gradual increase of the baryon number density starting at the end of the plateau suggests a continuous transition to the quark matter. The comparison with the RHMC results for the phase quenched model reveals a clear difference in the case of $m=0.01$. These results encourage us to increase the lattice size further. Simulations on a $16^{4}$ lattice are on-going.

\section{Acknowledgements}

This research was supported by MEXT as "Priority Issue on Post-K computer" (Elucidation of the Fundamental Laws and Evolution of the Universe) and Joint Institute for Computational Fundamental Science (JICFuS). Computations were carried out using computational resources of the K computer provided by the RIKEN Advanced Institute for Computational Science through the HPCI System Research project (Project ID:hp180178). J. N. was supported in part by Grant-in-Aid for Scientific Research (No. 16H03988) from Japan Society for the Promotion of Science. S. S. was supported by the MEXT-Supported Program for the Strategic Research Foundation at Private Universities "Topological Science" (Grant No. S1511006).

\section{References}

[1] J. R. Klauder, Phys. Rev. A29, 2036 (1984).

[2] G. Parisi, Phys. Lett. 131B, 393 (1983).

[3] G. Aarts, E. Seiler, and I.-O. Stamatescu, Phys. Rev. D81, 054508 (2010), arXiv:0912.3360.

[4] J. Nishimura and S. Shimasaki, Phys. Rev. D92, 011501 (2015), arXiv:1504.08359.

[5] K. Nagata, J. Nishimura, and S. Shimasaki, Phys. Rev. D94, 114515 (2016), arXiv:1606.07627.

[6] G. Aarts, F. A. James, E. Seiler, and I.-O. Stamatescu, Phys. Lett. B687, 154 (2010), arXiv:0912.0617.

[7] E. Seiler, D. Sexty, and I.-O. Stamatescu, Phys. Lett. B723, 213 (2013), arXiv:1211.3709.

[8] Y. Ito and J. Nishimura, JHEP 12, 009 (2016), arXiv:1609.04501.

[9] G. Aarts, L. Bongiovanni, E. Seiler, D. Sexty, and I.-O. Stamatescu, Eur.Phys.J. A49, 89 (2013), arXiv:1303.6425.

[10] G. Aarts, F. Attanasio, B. Jäger, and D. Sexty, JHEP 09, 087 (2016), arXiv:1606.05561.

[11] D. Sexty, Phys. Lett. B729, 108 (2014), arXiv:1307.7748.

[12] Z. Fodor, S. D. Katz, D. Sexty, and C. Török, Phys. Rev. D92, 094516 (2015), arXiv:1508.05260.

[13] K. Nagata, J. Nishimura, and S. Shimasaki, (2018), arXiv:1805.03964.

[14] D. K. Sinclair and J. B. Kogut, EPJ Web Conf. 175, 07031 (2018), arXiv:1710.08465.

[15] J. B. Kogut and D. K. Sinclair, Phys. Rev. D66, 034505 (2002), arXiv:hep-lat/0202028. 